\title{
Elucidation of the
} Mechanism of Gene Silencing using Small Interfering RNA:DNA Hybrid Molecules

L. C. Dugan

February 10, 2006 


\section{Disclaimer}

This document was prepared as an account of work sponsored by an agency of the United States Government. Neither the United States Government nor the University of California nor any of their employees, makes any warranty, express or implied, or assumes any legal liability or responsibility for the accuracy, completeness, or usefulness of any information, apparatus, product, or process disclosed, or represents that its use would not infringe privately owned rights. Reference herein to any specific commercial product, process, or service by trade name, trademark, manufacturer, or otherwise, does not necessarily constitute or imply its endorsement, recommendation, or favoring by the United States Government or the University of California. The views and opinions of authors expressed herein do not necessarily state or reflect those of the United States Government or the University of California, and shall not be used for advertising or product endorsement purposes.

\section{Auspices Statement}

This work was performed under the auspices of the U. S. Department of Energy (DOE) by the University of California, Lawrence Livermore National Laboratory (LLNL) under Contract No. W-7405-Eng-48. The project (03-ERI-004) was funded by the Laboratory Directed Research and Development Program at LLNL. 


\title{
FY05 LDRD Final Report Elucidation of the Mechanism of Gene Silencing using Small Interfering RNA:DNA Hybrid Molecules LDRD Project Tracking Code: 03-ERI-004 Lawrence Dugan, Principal Investigator
}

\begin{abstract}
The recent discovery that short hybrid RNA:DNA molecules (siHybrids) induce longterm silencing of gene expression in mammalian cells conflicts with the currently hypothesized mechanisms explaining the action of small, interfering RNA (siRNA). As a first step to elucidating the mechanism for this effect, we set out to quantify the delivery of siHybrids and determine their cellular localization in mammalian cells. We then tracked the segregation of the siHybrids into daughter cells after cell division. Markers for siHybrid delivery were shown to enter cells with and without the use of a transfection agent. Furthermore, delivery without transfection agent only occurred after a delay of 2-4 hours, suggesting a degradation process occurring in the cell culture media. Therefore, we studied the effects of nucleases and backbone modifications on the stability of siHybrids under cell culture conditions.
\end{abstract}

\section{Introduction/Background}

The silencing of specific genes by double stranded RNA molecules that are homologous to the sequence of the gene was first described in Caenorhabditis elegans by Fire and Mello and termed RNA inteference (RNAi) [1]. Furthermore, it was shown that this process was similar to a process in plants known as posttranscriptional gene silencing (PTGS) [reviewed in 2] and that it plays important roles, including antiviral defense, genome protection and developmental regulation in plant and mammalian species [reviewed in 3]. Recently, it was shown that the introduction of short, double-stranded (21-23 base oligomer) RNA molecules into mammalian cells could also cause sequence-specific mRNAdegradation [4]. These short, interfering RNA (siRNA) molecules can thus be used in a post-transcription mediated process to silence gene function. However, the effect of this process is short-lived and on the order of days.

The mechanism of action of RNA interference involves the identification of doublestranded (ds)RNA by the enzyme DICER (a homologue of E. coli RNase III). Dicer then digests the dsRNA into 21-23 nt dsRNAs containing 2-3 nt overhangs at the 3' ends, known as small interfering (si)RNAs. These siRNAs are then incorporated into

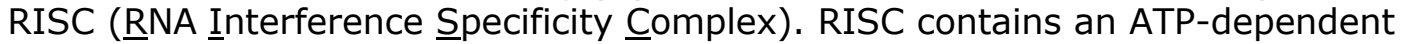
helicase, which unwinds siRNA, allowing the antisense strand (RNAa) of the siRNA to bind to its homologous mRNA sequence. RISC also contains a nuclease which cleaves the mRNA into fragments which are then digested by other cellular RNases [reviewed in 3,5$]$.

Recently, it has been demonstrated that varying the composition of siRNA molecules from RNA:RNA to RNA:DNA (siHybrid) molecules provided a similar gene silencing effect as siRNA [6]. The results from this work indicated that the greatest effect was produced when the siHybrid molecule was made up of an RNA sense strand (RNAs) 
and an antisense DNA strand (DNAa). Therefore, the mechanism described above for the action of siRNA molecules, using the RNAa strand as the target does not explain the results shown in the paper by Lamberton and Christian.

In order to fully understand the mechanism of how siHybrid molecules lead to silencing of gene expression and to further develop this methodology, it is important to first answer a number of obvious questions. These include: do the hybrid molecules reside and function within the cytoplasm or nucleus after entering the cell, how many of the molecules are required to induce the effect and what is their halflife within the cell. Do the siHybrids target complementary mRNA in the same manner as siRNA, or is there another target, protein translation, for example. Finally, can we identify any proteins that play a role in this process? Answers to these questions will provide important information regarding this technology for future research and possible clinical uses.

\section{Research Activities}

The specific research aims for elucidating the mechanism of action of siHybrid molecules are as follows:

Quantification of Bromine (Br)-labeled siHybrid molecules using PIXE. We set out to quantify the delivery of siHybrid molecules by aided and unaided transfection using Proton-induced X-ray emission (PIXE) analysis. PIXE is an accurate, absolute, fully quantitative technique that produces sample elemental concentrations from the $x$-ray yield induced by proton bombardment. It does not however, provide spatial information on the samples. PIXE experiments utilized internal labeling with bromodeoxyuridine and bromouridine on the DNA and RNA strands, respectively. Furthermore, as a comparison, siRNA was also labeled with Bromine on one strand. Cell cultures were then dosed with Bromine labeled siHybrids and siRNA, cultured overnight, rinsed and incubated an additional 24 hours. Cells were then collected, rinsed and analyzed for $\mathrm{Br}$ content by PIXE.

\section{Cellular localization of fluorochrome-labeled siHybrid molecules. To}

determine where in the cells siHybrids function, we set out to track the localization of siHybrid molecules following unaided transfection using fluorescence imaging. siHybrid molecules were either tagged on the $5^{\prime}$ ends of the DNA and RNA strands with $\mathrm{Cy} 5$ and $\mathrm{Cy} 3$ fluorochromes. Cells were dosed with labeled siHybrids and imaged over the course of 8-12 hours.

Cellular localization of Bromine-labeled labeled siHybrid molecules. To further determine the cellular location in which siHybrids function, we exposed cells to bromine tagged siHybrids, collected the cells following our standard protocol and then imaged flash-frozen lyophilized cells using the NanoSIMS instrument.

Effects of nucleases on siHybrid stability. A delay in unaided delivery of labeled siHybrids was observed in both the PIXE and fluorescence studies. This led us to suspect that degradation was occurring in the cell culture media and that it was due to nucleases present in the serum. DNA oligomers 4-6 nucleotides in length are known to be readily taken into mammalian cells. Therefore, we felt it was possible that our siHybrids (23 nucleotides in length) were being digested down to this length prior to entering the cells. We tested this hypothesis by measuring the degradation of siHybrids incubated in media with and without serum and with several 
recombinant nucleases. Furthermore, the cellular uptake of shortened siHybrids, 6 nucleotides in length was compared to the standard 21 nucleotide long siHybrid.

Effects of backbone modifications on stability of siHybrids. Several modifications to the base composition of oligonucleotides have been shown to stabilize RNA and DNA and siRNA in the presence of nucleases. Some have further shown improvements in the level of response of cells to siRNA-mediated gene silencing. To test the effect of these modifications on our system, we designed siHybrids containing a number of these alterations and looked at stability in the presence of serum nucleases in vitro and at the effect on gene silencing in cells.

\section{Results}

Quantification of Bromine-labeled siHybrid molecules using PIXE. siHybrids and siRNA tagged with Bromine were transfected or delivered unaided in Hela cell cultures containing $\sim 10^{6}$ cells. Cells were collected and analyzed by PIXE and results are shown in Figure 1. Transfection of $40 \mathrm{nM}$ solution of siHybrids or siRNA yielded an average of $\sim 1-1.5 \times 10^{7} \mathrm{Br}$-tagged molecules per cell (Figure $1 \mathrm{~A}$ ). Unaided delivery of $\mathrm{Br}$-siRNA did not lead to detectable levels of $\mathrm{Br}$ in the cells. Unaided delivery of $\mathrm{Br}$-siHybrids led to a dose dependent level of $\mathrm{Br}$ in the cells. At a dose of $40 \mathrm{nM}$ (that used in transfection) we measured $\sim 1 / 2$ the level of $\mathrm{Br}$ in the cells compared to transfected siHybrids. We did not detect a plateau effect at the doses used in these experiments. Results shown are for measuring two or three sample spots for each of two cultures from two independent experiments and error bars represent the standard deviation for the measurements (minimum of 6 spots measured for each data point). We next looked at the uptake of unaided delivery of siHybrids over time by dosing dishes containing $\sim 10^{6}$ Hela cells with $120 \mathrm{nM}$ of $\mathrm{Br}-$ siHybrid. Samples were collected, rinsed and spotted at increasing timepoints. Results for siHybrids tagged with $\mathrm{Br}$ on the sense or antisense strand are shown in Figure 1B. It is clear from these measurements that there is a delay of $\sim 2-4$ hours before significant levels of $\mathrm{Br}$ are present in the cells.

Figure 1. Quantification of Bromine by PIXE. Levels of Br-tagged siHybrids and siRNA in cells were measured by PIXE. Aided and unaided delivery were compared as was dose for unaided siHybrids (A). The cellular uptake of $\mathrm{Br}$-tagged siHybrids was also measured over the course of a day (B). 

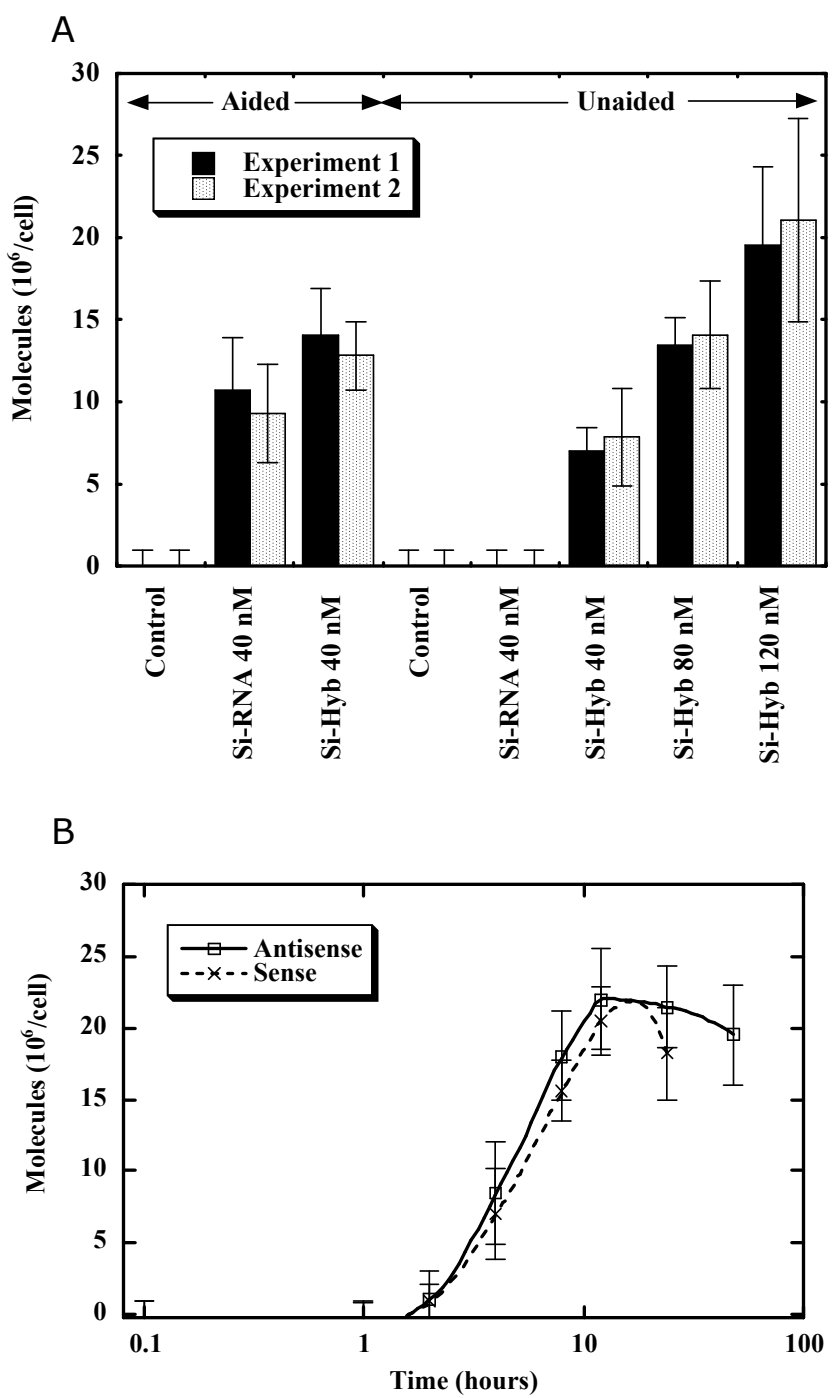

Cellular localization of fluorochrome-labeled siHybrid molecules. SiHybrids are thought to function in the cytoplasm and to target mRNA prior to it's translation to protein, which occurs in the ribosomes. Ribosomes localize on the cytoplasmic surface of the rough endoplasmic reticulum ( $r E R$ ), an organelle that is continuous with the nuclear envelope and the cytoplasmic surface of the nuclear membrane. Localization of siHybrids to this region would suggest that they target mRNA at the ribosomes. However, if siHybrids localized in the nucleus, it would suggest that the target was pre-mRNA or DNA. Hela cell cultures growing in glass-bottom tissue culture dishes were dosed with fluorochrome-labeled siHybrids with (aided) and without (unaided) transfection reagent. Cells were then imaged at various times after exposure. Aided delivery resulted in localization of siHybrids in the perinuclear region of the cell, which includes the Golgi complex, mitochondria and the (ER), $24 \mathrm{~h}$ after transfection (A). Unaided delivery also resulted in localization of siHybrid signal in the perinuclear region, but a significant portion of the signal was also localized in mitochondria spread throughout the cell during the same $24 \mathrm{~h}$ timeframe. This dispersed signal may partially explain the reduced and sporadic gene silencing results we see with unaided delivery of siHybrids. 
PIXE results indicated there was a delay in uptake of $\mathrm{Br}$-siHybrids. To confirm these results we dosed Hela cells with fluorochrome-labeled siHybrids and imaged them over the course of $24 \mathrm{~h}$. No fluorochrome was detectable immediately or $2 \mathrm{~h}$ after dosing. However, at $4 \mathrm{~h}$ nearly all cells had some fluorescence signal. Fluorescence intensity continued to increase through the imaging period $(24 \mathrm{~h})$. These experiments, illustrated in Figure 3 confirm the delay in uptake previously detected by PIXE.

To determine if siHybrids have the ability to reduce gene expression over multiple generations, we studied the segregation of fluorochrome-labeled siHybrids in the daughter cells of dosed cells. As shown in Figure 4, significant fluorochrome signal was present in cells even after 10 days.

Finally, to help understand if siHybrids were being degraded to a shorter, more cell permeable length, we dosed cells with reduced length siHybrids. These constructs were prepared with 6 nucleotide RNA and DNA strands, instead of the usual 21-23 nucleotide strands. Mammalian cells have been shown to be permeable to short DNA oligomers on the order of 4-6 nucleotides in length, although not to RNA oligomers. If nucleases in the cell culture media were digesting the siHybrids, they might reach a minimum length that was permeable to cell membranes. Short siHybrids would be expected to be readily detected in cells after unaided delivery. As shown n Figure 5, shorter siHybrids were detected in cells within $1 \mathrm{~h}$ of dosing, while standard length siHybrids were not detected until $3 \mathrm{~h}$ after dosing.

Figure 2. Microscopic tracking of aided and unaided siHybrid transfection in Hela cells. Aided delivery (A) and unaided delivery (B) of Cy3-labeld siHybrids in Hela cells. Cells were imaged on an inverted fluorescence microscope equipped with phase contrast. Phase contrast and fluorescence images were overlaid and fluorescence image layers were psuedocolored. Black and white image of unaided Cy3-labeled siHybrids in Hela cells (C) clearly shows strong mitochondrial localization of fluorescent signal. Scale bar in A and B is $30 \mu \mathrm{m}$.

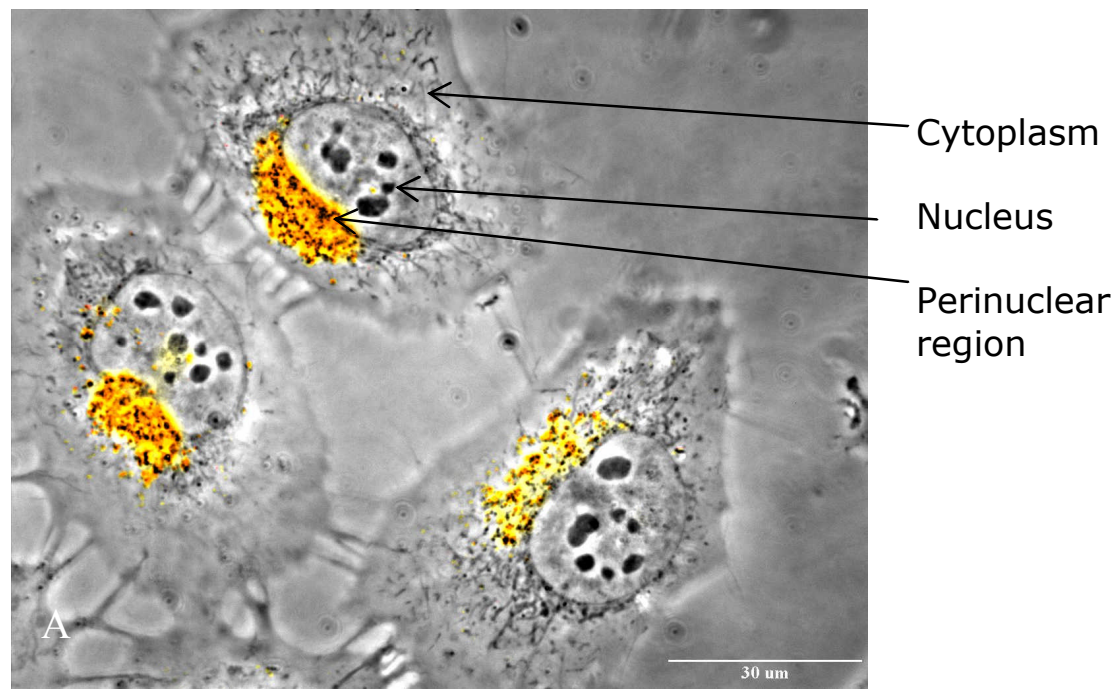



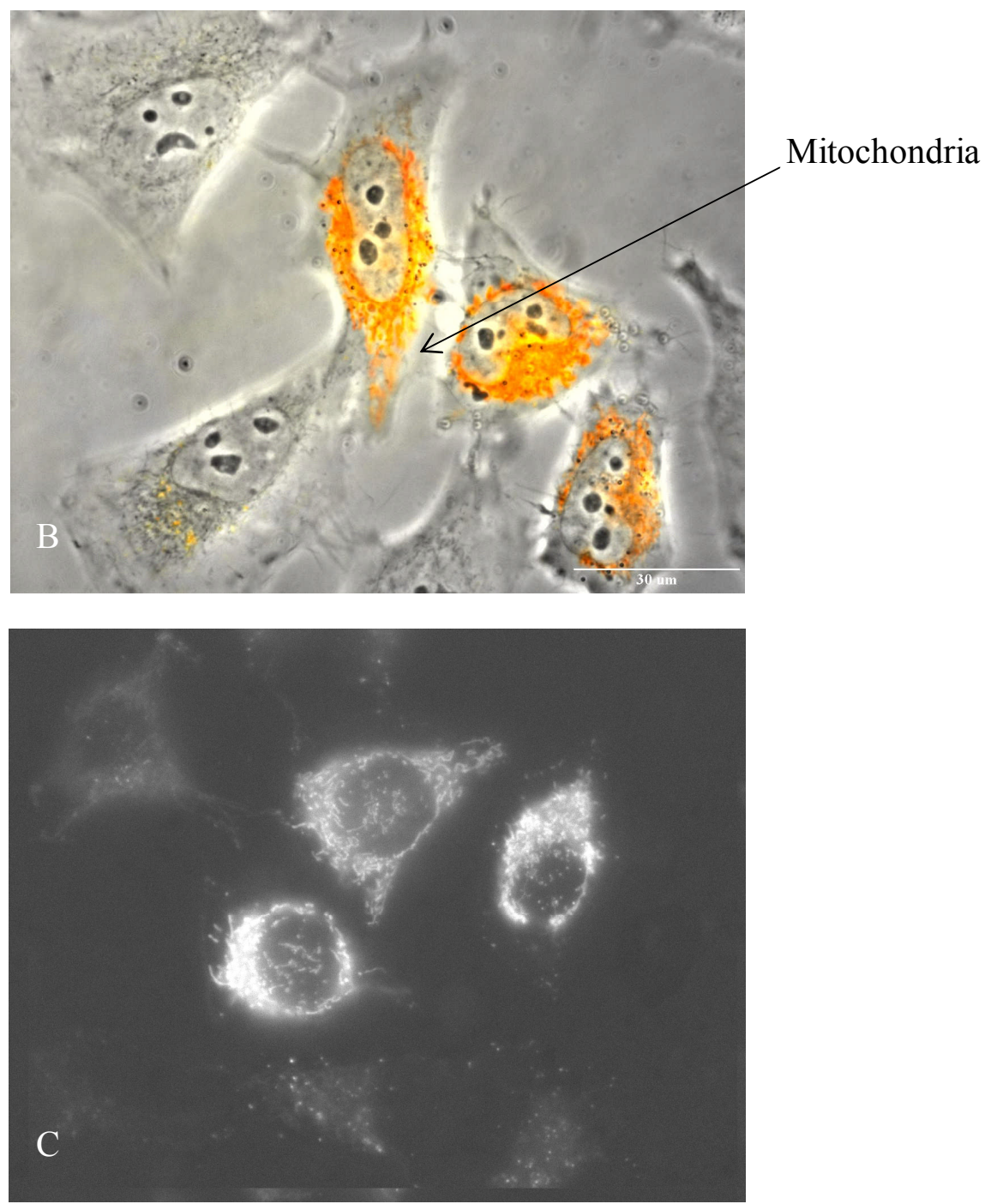
Figure 3 Delayed uptake of unaided siHybrids. Hela cells growing in glass bottom dishes were dosed with Cy5-labeled siHybrids. Images were taken at $2 \mathrm{~h}$ intervals after addition of siHybrids to cell culture media. Cy5 signal is shown psuedocolored in red.
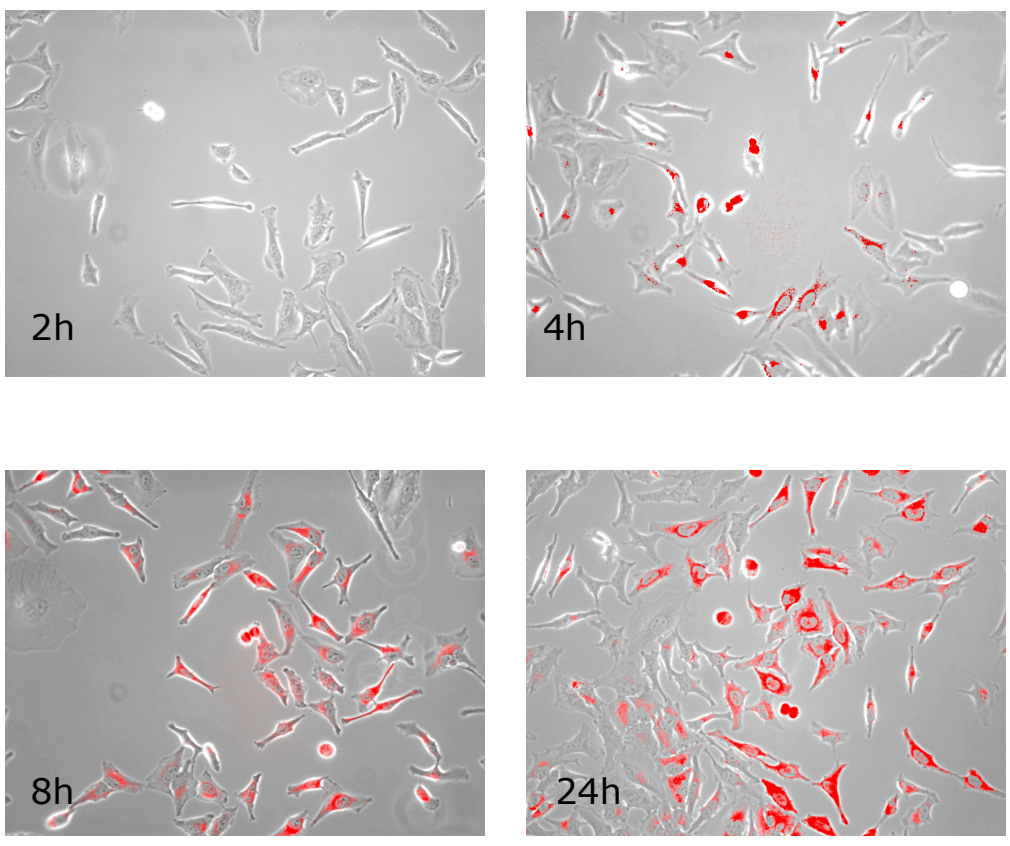
Figure 4. Segregation of siHybrids to daughter cells during cell division. Hela cells were plated at a low density on gridded glass bottom dishes and dosed with Cy5-siHybrids. The grid allowed for tracking initial cells through multiple generations. Individual cells were then imaged along with clonal expansions of those cells. The presence of the Cy5 signal is again pseudocolored in red.
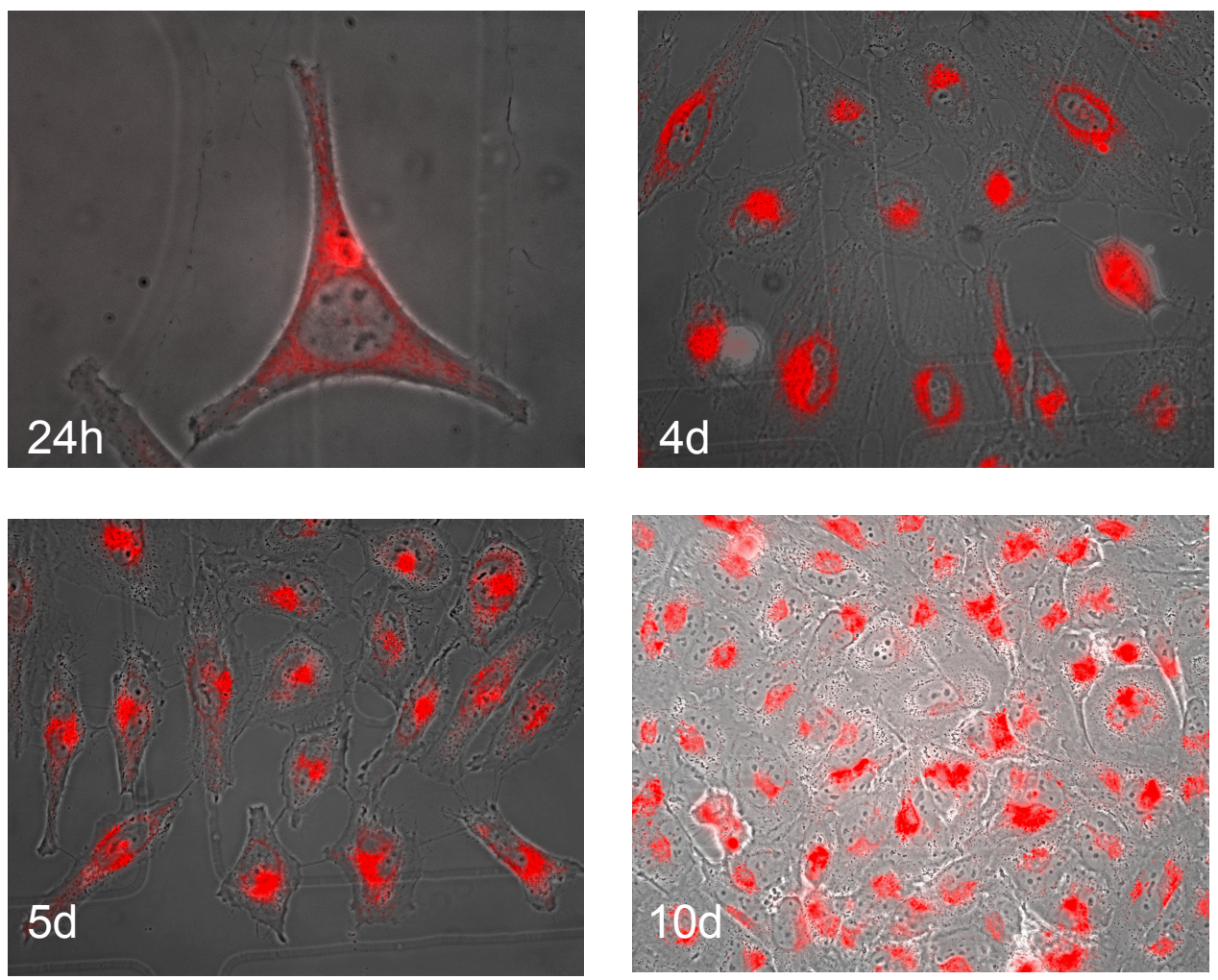
Figure 5. Uptake of short (6 nucleotide) siHybrids. Cy5-labeled siHybrids were constructed with 6 nucleotides strands and standard 21 nucleotide strands. Hela cells on glass bottom dishes were then exposed to $40 \mathrm{nM}$ concentrations of these constructs. Images were taken over a $24 \mathrm{~h}$ period. Short, 6 nucleotide siHybrids are shown on the left, while standard length, 21 nucleotide sihybrids, are shown on the right. Cy5 signal was not pseudocolored in these images, but appears as intense white pixels.
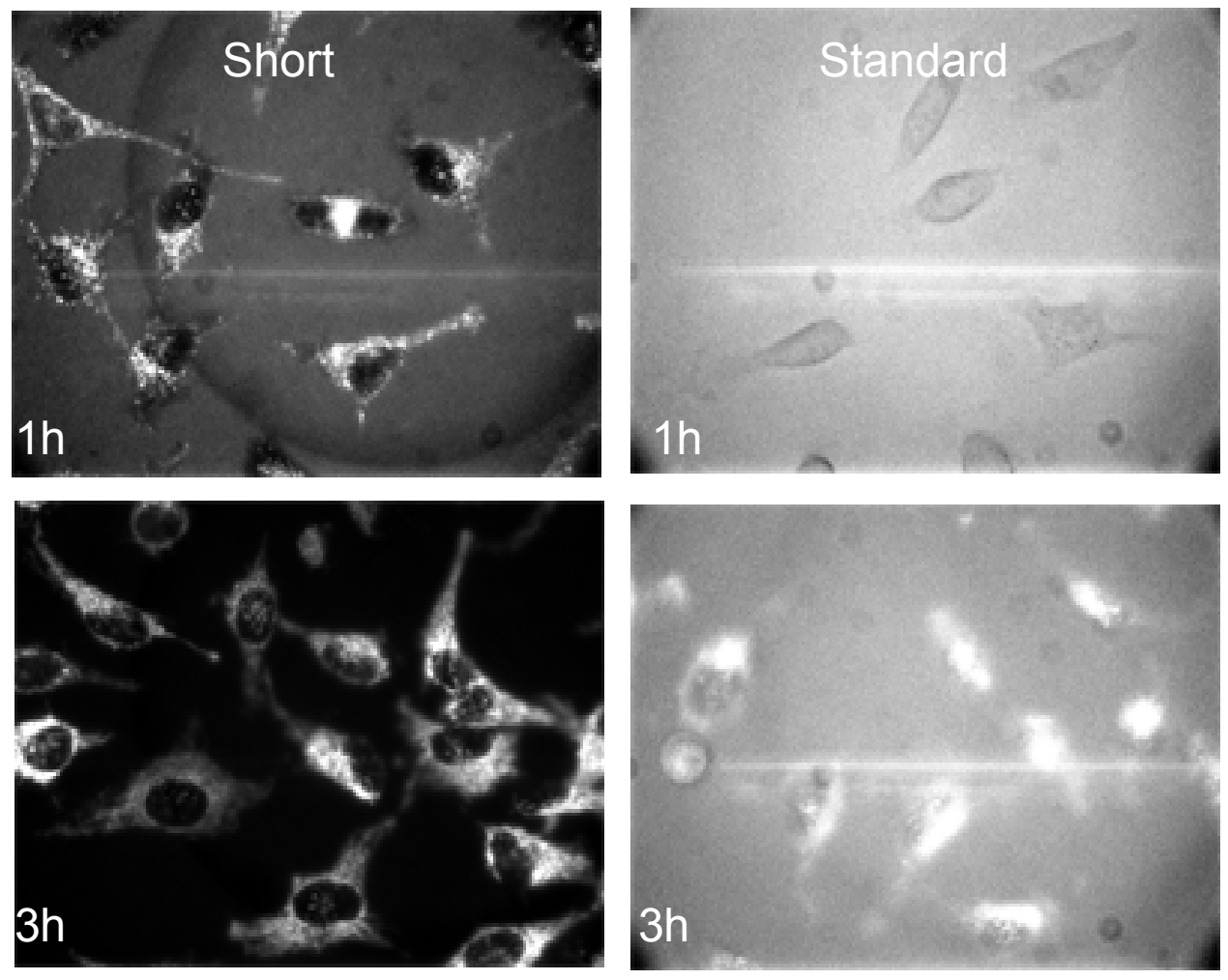

\section{Cellular localization of Bromine-labeled labeled siHybrid molecules.}

Cleavage of the fluorochrome label from the backbone of the siHybrid may have occurred during unaided delivery of siHybrids. This in turn may have lead to false localization results which indicate the siHybrids were segregated into the mitochondria. NanoSIMS provides an analytical tool which is capable of quantifying ions and spatial localization down to $50 \mathrm{nM}$ resolution. A preliminary NanoSIMS experiment was performed to determine if $\mathrm{Br}$-labeled siHybrids localized to the nucleus or cytoplasm, the results of which are shown in Figure 6. After dosing with $\mathrm{Br}$-siHybrids, the cells were incubated for $24 \mathrm{~h}$, flash-frozen and lyophilized. Bromine can be seen at high levels in the nucleus of several cells (bottom right panel Figure $6 \mathrm{~A}$, right panel Figure 6B). Bromine was also detected in the spaces between the cells (Figure 6B, right) and scattered at low levels in the cytoplasm. Degradation of the siHybrids by nucleases would allow for the recycling of the nucleotides by cells, leading to their incorporation in DNA by salvage pathways. This would produce an expected Bromine signal in the nucleus, as bromodeoxyuridine and bromouridine are often used to label nuclear material in cell cycle studies. These results further suggest a degradative effect of exposure of the siHybrids to nucleases present in the cell culture media. A comparison of aided and unaided delivery of Br-siHybrids and Br-siRNA using NanoSIMS would likely provide additional insight into this 
phenomenon. Unfortunately, due to personnel and financial constraints, we were unable to further investigate this line of research.

Figure 6. NanoSIMS imaging of Hela cells transfected with Br-siHybrids. Nitrogen, phosphorous, carbon, chlorine and bromine spectra were taken of Hela cells dosed with $\mathrm{Br}$-siHybrids. Individual ion spectra are shown here for a region of cells and a closer scan of a single cell. An overlay of the $\mathrm{Br}$ spectra on chlorine spectra clearly shows the strong localization of $\mathrm{Br}$ in the nucleus and in the spaces between cells.
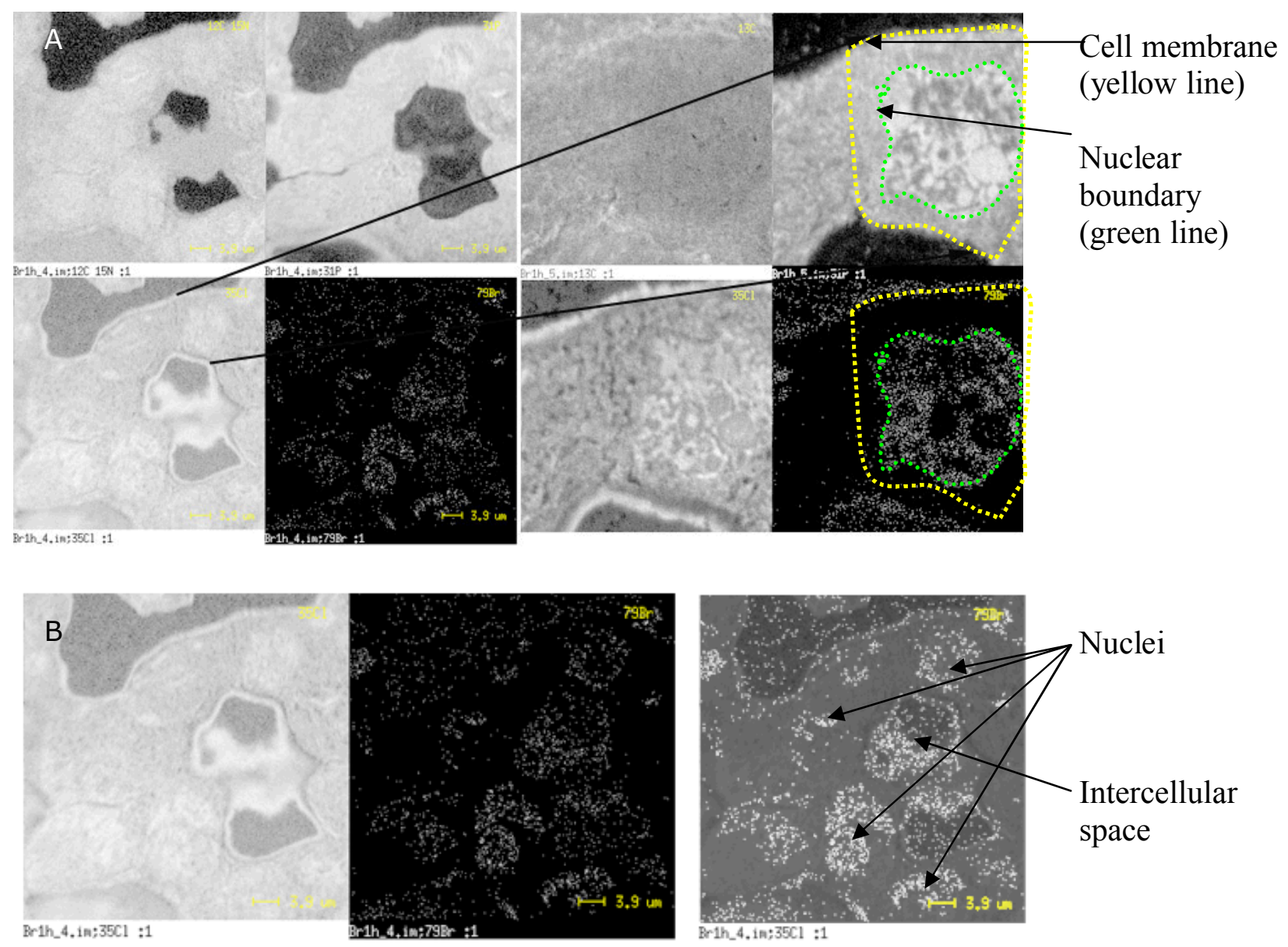

\section{Effects of nucleases on siHybrid stability}

We next set out to further understand the potential degradative effect of nucleases on unaided siHybrid delivery. SiHybrids were incubated in cell culture media containing $10 \%$ fetal bovine serum, a common component of media. It is evident from Figure 7 that siHybrids are readily degraded by nucleases in the media within the first $4 \mathrm{~h}$ of incubation. This was further confirmed by incubating in serum alone (data not shown). It has been shown that modification of DNA and RNA backbones can improve their stability in the presence of nucleases. To test if this held for siHybrids we designed constructs with modifications to both the RNA and DNA sequences. The addition of a methyl group on the $2^{\prime} \mathrm{OH}$ of RNA bases is known to prevent degradation of RNA by RNases. As shown if Figure 8, 2'OMe RNA antisensemodified siHybrids were stable when incubated in serum. However, they did not 
function to reduce gene expression in cells (data not shown). Modification of DNA oligomers with phosphorothioate backbones has been shown to increase DNA stability. siHybrids constructed with this modification on the DNA sequence did function when transfected into cells, but were no more stable in cell culture media than the unmodified sequences (data not shown).

Figure 7. Sihybrid stability in cell culture media. containing $10 \%$ serum (DF10). 5 ug siHybrid was incubated at 37C in 95 ul 10 mM Tris-HCl (left) or DF10 media (right). Digestion by extracellular nucleases was quenched by incubation at $65 \mathrm{C}$ for 20 min. Aliquots were then run on 1.5-2\% agarose gel, stained with $\mathrm{EtBr}$ and imaged. Images were scanned as 8bit TIFF files and analyzed using Metamorph's 1D gel analysis software. Results were graphed as the \% of siHybrid remaining in media at a given timepoint compared to incubation in Tris.

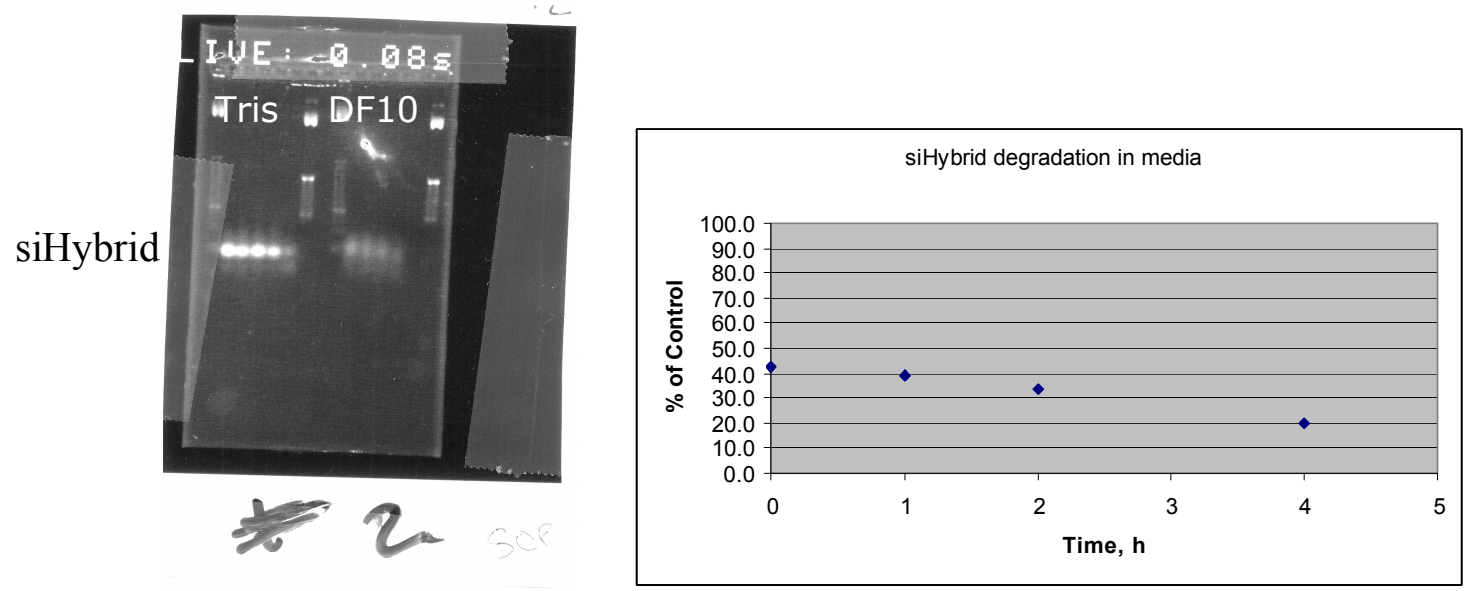

Figure 8. Enhanced stability of modified siHybrids. 2'OMe-RNA modified sihybrid degradation in cell culture media containing 10\% serum (DF10). 5 ug 2'OMe-RNA modified siHybrid was incubated at 37C in 95 ul $10 \mathrm{mM}$ Tris- $\mathrm{HCl}$ (left) or DF10 media (right). Digestion by extracellular nucleases was quenched by incubation at $65 \mathrm{C}$ for $20 \mathrm{~min}$. Aliquots were then run on 1.5-2\% agarose gel, stained with $\mathrm{EtBr}$ and imaged. Images were scanned as 8bit TIFF files and analyzed using Metamorph's 1D gel analysis software. Results were graphed as the \% of siHybrid remaining in media at a given timepoint compared to incubation in Tris.
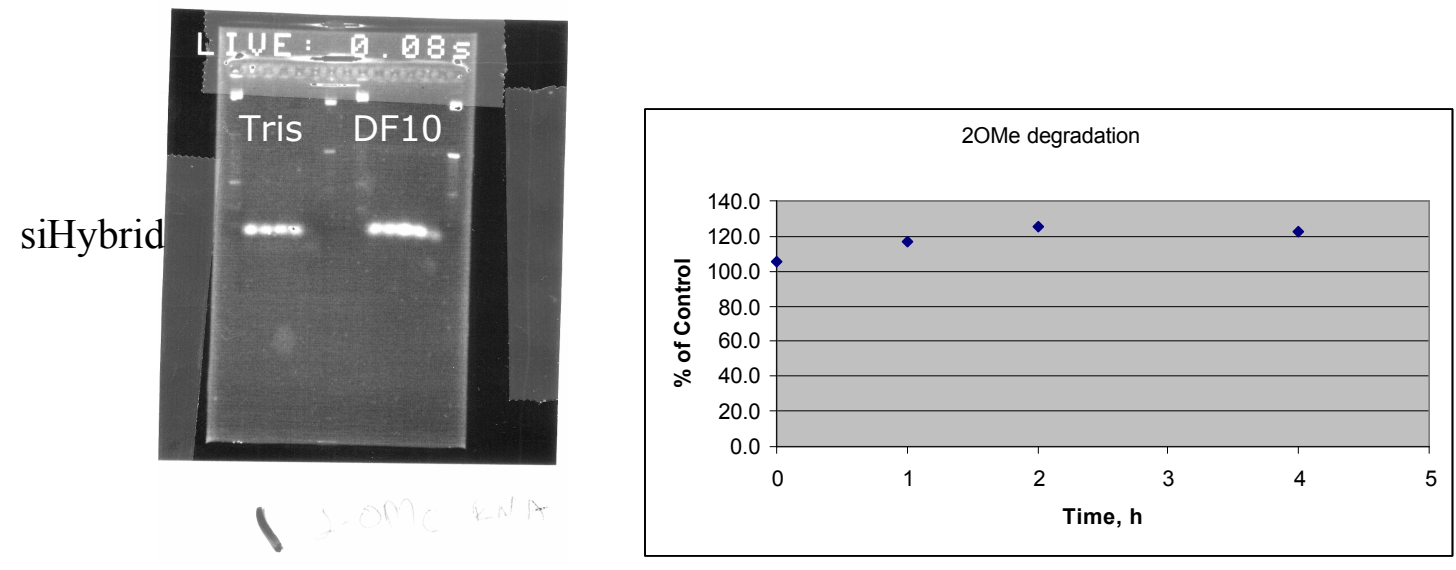


\begin{abstract}
Summary
siHybrids have been shown to reduce gene expression by sequence-specific targeting followed by nuclease digestion of complementary mRNA, similar to siRNA (see attached report UCRL-JRNL-209119 DRAFT). In this project we have quantified the average number of molecules transfected into a cell, shown that at least the tag $(\mathrm{Br}$ or fluorochrome) of labeled siHybrids can be measured in cells and by multiple techniques. Transfected siHybrids localize to the perinuclear region of cells, where they are in close proximity to their target molecule, mRNA. However, when siHybrids are delivered without the protective transfection reagent, nucleases present in the cell culture media (from the added serum) digest the sihybrids, leading to altered localization of the signal. This process takes $2-4 \mathrm{~h}$ in order for a signal to be detected. Finally, although we were able to improve the stability of siHybrids to attack by nucleases, we were unable to design a modification that maintained a similar level of function as unmodified constructs.
\end{abstract}

Acknowledgements (if applicable) The author would like to acknowledge Peter Weber for his assistance with the NanoSIMS imaging and Graham Bench for his assistance with the PIXE analysis.

\title{
References
}

1. Fire, A., et al., Potent and specific genetic interference by double-stranded RNA in Caenorhabditis elegans. Nature, 1998. 391(6669): p. 806-11.

2. McManus, M.T. and P.A. Sharp, Gene silencing in mammals by small interfering RNAs. Nat Rev Genet, 2002. 3(10): p. 737-47.

3. Baulcombe, D., RNA silencing. Curr Biol, 2002. 12(3): p. R82-4.

4. Elbashir, S.M., et al., Duplexes of 21-nucleotide RNAs mediate RNA interference in cultured mammalian cells. Nature, 2001. 411(6836): p. 494-8.

5. Kitabwalla, M. and R.M. Ruprecht, $R N A$ interference--a new weapon against HIV and beyond. N Engl J Med, 2002. 347(17): p. 1364-7.

6. Lamberton, J.S., Christian, Allen T., Varying the Nucleic Acid Composition of siRNA Molecules Dramatically varies the Duration and Degree of Gene Silencing. Mol Biotechnol, 2003. 24(2): p. 111-119. 